\title{
Wireless water quality monitoring system for high density aquaculture application
}

\author{
F. A. Saparudin', T. C. Chee' , A. S. Ab Ghafar ${ }^{3}$, H. A. Majid4, N. Katiran ${ }^{5}$ \\ 1,2,3,4Faculty of Engineering Technology, Universiti Tun Hussein Onn Malaysia, Malaysia \\ ${ }^{5}$ Faculty of Electrical and Electronic Engineering, Universiti Tun Hussein Onn Malaysia, Malaysia
}

\begin{tabular}{l}
\hline Article Info \\
\hline Article history: \\
Received Sep 27, 2018 \\
Revised Nov 25, 2018 \\
Accepted Dec 9, 2018 \\
\hline
\end{tabular}

Keywords:

Internet of things

Water quality monitoring

Wireless sensor

\begin{abstract}
Water quality is one of the major factors that greatly affects growth and mortality rate of aquatic livestock especially in high density aquaculture system. Conventional method requires fish farmer to perform manual water quality test and record on multiple fish tanks in regular basis. This process is meticulous, and may affect aquatic livestock that needs close and immediate attention. In this paper, water quality monitoring system for the high density aquaculture environment is proposed. The monitoring system is composed of multiple sensor nodes and sensor/server node hybrid, which used to collect and manage the water quality parameter data of multiple tanks. The sensor nodes collect and store the water quality parameters in local database and transmit them to the server node through a wireless communication. The server node is used for data analysis, processing and allow public access via web browser through various Wi-Fi enabled smart devices. This paper presents a proof-of concept of clustered wireless monitoring system focused on the use of multiple sensor nodes to determine the water parameter in real time. This system is cost effective, quickly deployable, and user-friendly with wireless capabilities.
\end{abstract}

Copyright @ 2019 Institute of Advanced Engineering and Science. All rights reserved.

\section{Corresponding Author:}

F. A. Saparudin,

Faculty of Engineering Technology,

Universiti Tun Hussein Onn Malaysia,

KM 1, Jalan Panchor, 84600 Pagoh, Johor, Malaysia.

Email: faizs@uthm.edu.my

\section{INTRODUCTION}

Aquaculture is increasingly considered as an integral component in the search for global world food security and economic development. This implies aquaculture requires intensive care in the rearing process to enhance production, including stocking, feeding, protection from predators and disease [1]. Regular care and maintenance are required to ensure the water quality is suitable for aquaculture. The water quality can affect the growth and may cause the death of the aquatic livestock. Thus an aquaculture system needs to provide suitable water quality to promote growth, control disease and reduce mortality. Especially in high density aquaculture, where a large number of aquatic livestock is raised in artificial tanks require a systemic approach to alleviate the complexity of managing the water quality manually.

In many of the developing and developed regions of the world, the demand for aquaculture products has continued to grow. In recent report, more fish for human consumption have originated from farms than from wild capture according to the latest global report from the Food and Agriculture Organization of the United Nations (FAO) [2]. The aquaculture sector is increasing in demand, one of the issues faced by the aquaculture sector is to be informed on water quality parameters of farmed aquaculture tanks. Especially in high density aquaculture, there are multiple tanks with rapid change in water quality need to be tested and recorded every day. Since this traditional method is not continuous and real time, the negligence may affect the aquatic livestock especially the sensitive ones. 
Therefore, there is a need for continuous and real time water quality monitoring system. The automation of aquaculture systems will allow the industry to improve environmental control, reduce catastrophic losses, reduce production cost, and improve product quality. The most important parameters to be monitored and controlled in an aquaculture system include temperature, dissolved oxygen, $\mathrm{pH}$, ammonia, nitrates, salinity, and alkalinity, since they directly affect animal health, feed utilization, growth rates [3]. This especially hard to maintain in a high density aquaculture where the water quality is inherently unstable. The survival and growth of aquatic livestock are critically depend on the water quality [4].

This work focuses on the uses of multiple microcomputer and sensors to monitor the water quality parameters in aquaculture in real-time. The monitoring system is composed of multiple sensor nodes, which used to determine and collect the water quality parameter of multiple tanks and transmit them to the server node Wi-Fi technology. The server node basically provides service such as sharing information or resources for other program or devices. The function of server node is centralized data collection, processing and presentation the information through a web server. The information can be accessed using any browser via Wi-Fi enabled smart devices on local network. The proposed aquaculture water monitoring system is suitable to be placed in high density aquaculture to simultaneous and effectively monitor multiple fish tanks.

\section{RELATED WORKS}

Water quality monitoring system has been widely studied and developed around the globe. Wireless sensor network is one of the approach enabling aquaculture environmental monitoring. A WSN based on Zigbee in aquaculture was presented by Wen-Tsai Sung \& Jui-Ho Chen. The system is network surveillance combined with mobile devices and a remote platform to collect real-time farm environment information. The real time data measures using A/D signal processing via ZigBee wireless transfer to the terminal server. The data messages are analytically processed and send to the cloud database and displayed on a computer terminal [5].

A water quality monitoring and control system for aquaculture based on wireless sensor networks and single chip computer technology as a base in the actual operation was conducted by Daudi S. Simbeye and Shi Feng Yang. The Sensor nodes collected the water quality parameters of temperature, dissolved oxygen, $\mathrm{pH}$ level and water level and transmitted them to the base station host computer through ZigBee. A short message sent to the owner via the Global System for Mobile (GSM) module for the notification when monitored variables take anomalous values [6].

In the meantime, B O'Flynn and co-workers had developed a water monitoring system names as Smart Coast. Smart Coast is an applied R\&D Project, co-funded by the Irish Marine Institute and EPA, which aims to develop WSN is featuring Plug and Play sensor platforms, novel sensors and low power consumption communication. The key parameters included temperature, phosphate, dissolved oxygen, conductivity, pH, turbidity and water level. The WSN deployment will enable in-situ chemical and physical measurements and allowed data to be viewed in real time through the internet by remote users [7].

A dynamic and smart wireless mesh sensor networks were designed and implemented for aquaculture and water quality management applications by John Adinya Odey and Li Dao Liang. The system utilized the Waspmote embedded system platform developed by Libelium, mesh networking transceiver from Digi International and Smart sensors from UNISM to implement a novel smart Wireless Mesh sensor network. The system was designed to continuously monitor aqua environmental parameters and then initiated an alert or early warning to system user when a certain threshold is exceeded. The data generated is stored locally on the gateway or sent to remote web server. Data can be accessed with smart mobile phones or personal computer [8].

Besides that, CDMA service combined with IPsec-based virtual private network (VPN) function also often used in aquaculture monitoring system. A remote wireless water quality monitoring system was developed in Intensive Fish Culture. The remote monitoring system used CDMA service combined with VPN function to construct a wireless sensing network in countrywide scale. The research integrated with a forecasting model on the basis of artificial neural network (ANN) to provide real-time information and the dynamical trend of the water quality at different monitoring sites. The detected data can be collected and analyzed at any time via internet and the system provided early warning [9]. In [10] a water quality dynamic monitoring system based on Web-Server-Embedded Technology for aquaculture. The web server embedded chip is used to obtain water quality information from different sensors. The communication module is composed of a Wi-Fi module and a CDMA module with VPN function for establishing wireless local area network and achieving remote communications and data transmission.

Moreover, the use of microcontroller and microprocessor is often seen in aquaculture. The measurement system developed by Jasman Bin Jaffar was built by Microcontroller ATMEL AT89S52 
with $\mathrm{pH}$ and temperature sensors together with Liquid Crystal Display (LCD) and keypad [11]. Apart from that, Sai Krisha Vaddadi and co-workers had developed a multiple water quality measurement system using a different sensor instrument, embedded controller and wireless communication device (XBee-Pro). A PIC $18 \mathrm{~F} 4520$ microcontroller was interfaced with the measurement unit to read and validates the measurement and stored the data in memory. In the meantime, the wireless network protocol was developed to maintain the harmonious communication between measurement devices and remote station [12].

A novel kind of smart monitoring system for aquaculture were developed by H.L Chen \& X.Q. Liu. This system used the Android mobile phone platform and GPRS communication technology to achieve the remote wireless monitoring on real significance. The management of water quality parameters included water level, $\mathrm{pH}$ level, dissolved oxygen and temperature. A 16-bit microprocessor MSP430f149 of TI company was adopted on acquisition part, 12 high-precision AD converter is used and also PID control was adopted on the control part. The sensor collected data through the GPRS module and sent to the remote server, and then the server sent to the Android mobile phone. In addition, the pond water quality parameters can be remotely monitored through a mobile phone [13].

From the literature, most of the proposed monitoring system still limited by its rather complicated operational requirements and high maintenance cost. The collected sensor data is usually stored in dedicated server facility that require cost. By adopting centralized sensor cluster topology as shown in Figure 1, it is possible to vastly interconnect multiple sensor nodes with on-site low cost server to handle light internal or web traffic. Grouping a set of microcomputer to work as a server is chiefly much more cost-effective than a normal server.

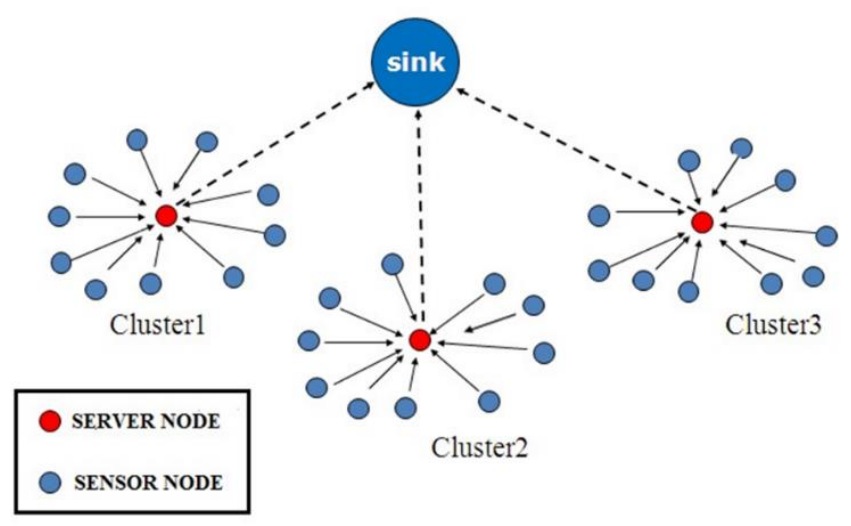

Figure 1. Centralized cluster sensor node topology

\section{SYSTEM ARCHITECHTURE}

Improving the hardware and software aspects from the previous works, the proposed water quality monitoring system consist of quickly deployable and cost-effective sensor nodes and server node. Water tmperature sensor sensors are used in this project only as a proof-of-concept. The system allows various other types of sensors such as Oxygen, ORP, Salinity, and more to be easily interchanged or added. The sensor node monitor and measure the parameter in real-timeand transmit the data wirelessly to a server node via WiFi communication. Figure 2 shows the network overview of the proposed water quality monitoring system. Multiple sensor nodes share their sensor data to a server/sensor node hybrid. The hybrid node is a server that can collect its own sensor data while collecting data from other sensor nodes. This can reduce the cost of having dedicated facilities for database and web server.

Then, user Wifi enabled devices/equipment including smartphones, laptop and PC can centrally access the data from the server node via web service provided by the server node. Note that in this architecture, sensor node can also be programmed to work simultaneously as server node. Therefore, this system does not require to have a dedicated device to act as server nor external server 


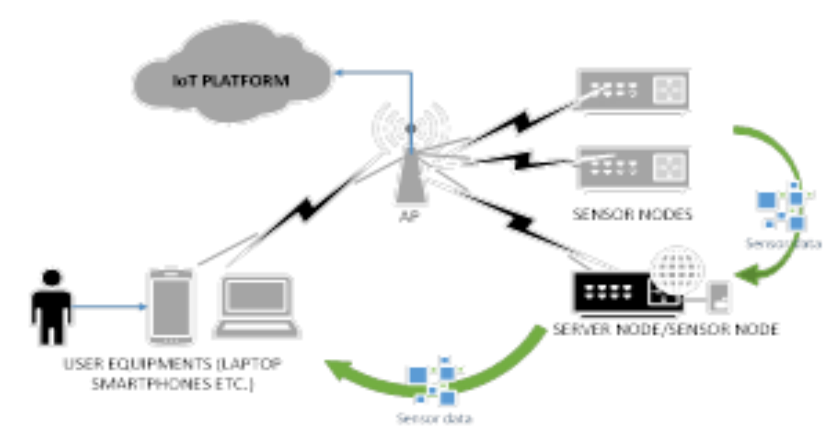

Figure 2. Network overview of the proposed wireless water quality monitoring system

\subsection{Sensor Node}

Sensor nodes is basically consists of various sensors which use to detect the predetermined parameters that indicates the water quality. There are virtually limitless no of sensors that can be integrated in a single sensor node. As a proof of concept, temperature sensor is used in this system. DS18B20 temperature sensor is interfaced with a microcomputer (i.e. Raspberry Pi) through the GPIO pins to detect the temperature. The water quality parameter are stored in a MySQL database and it is transferred to server node based on the SSH key-based authentication. SSH or secure shell is an encrypted protocol used to administer and communicate with servers. SSH keys provide an easy, yet extremely secure way of transferring the database into the server nodes. Apart from that, the temperature sensor used in the system is a pre-wired and waterproofed version of the DS18B20 sensor. The sensor is good up to $125^{\circ} \mathrm{C}$ but the cable is jacketed in PVC so the suggested temperature is under $100^{\circ} \mathrm{C}$. The temperature sensor are digital, they can prevent signal degradation even over long distances. These 1-wire digital temperature sensors are fairly precise $\left( \pm 0.5^{\circ} \mathrm{C}\right.$ over much of the range) and can give up to 12 bits of precision from the onboard digital-to-analog converter. Figure 3 shows the hardware and the circuit of the sensor node respectively.

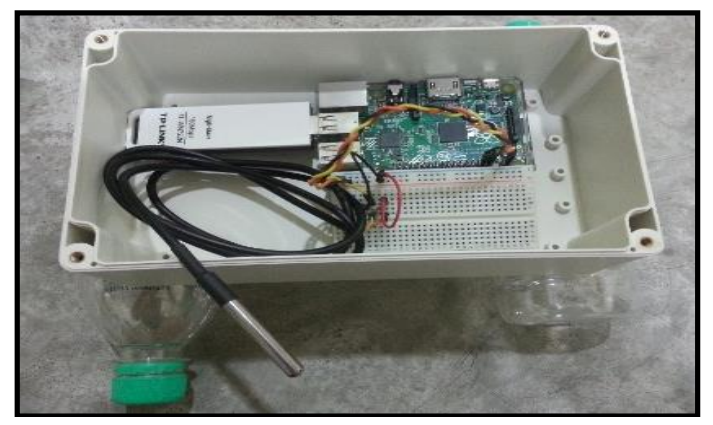

Figure 3. Hardware of sensor node

\subsection{Server Node}

In terms of hardware, Server Node is completely identical with sensor nodes. The key difference is that server node basically provides services for sharing data or resources to other program or devices in the network. The server node is designed to collect the water quality data or information from all sensor nodes in the network and deliver to the user as requested. Serve node stored the information transferred from the sensor nodes in a local MySQL database, process and transmit the data to a web server using the LAMP (Linux, Apache, MySQL, and PHP/Python) stack and WordPress application. The user can visit the web server by using any web browser. The communication between the user and server node takes place using the Hypertext Transfer Protocol (HTTP). Figure 4 shows the basic operation of sensor/server node. The system starts when the power if turned on. The program write in Python or PHP give the instruction to the sensors in order to measure the parameter. Then the information of the parameter stored in a local MySQL database of sensor nodes. Then the data transfer to the local MySQL database of server node and the data will fetched to a web server and it can be accessed via any web browser. 


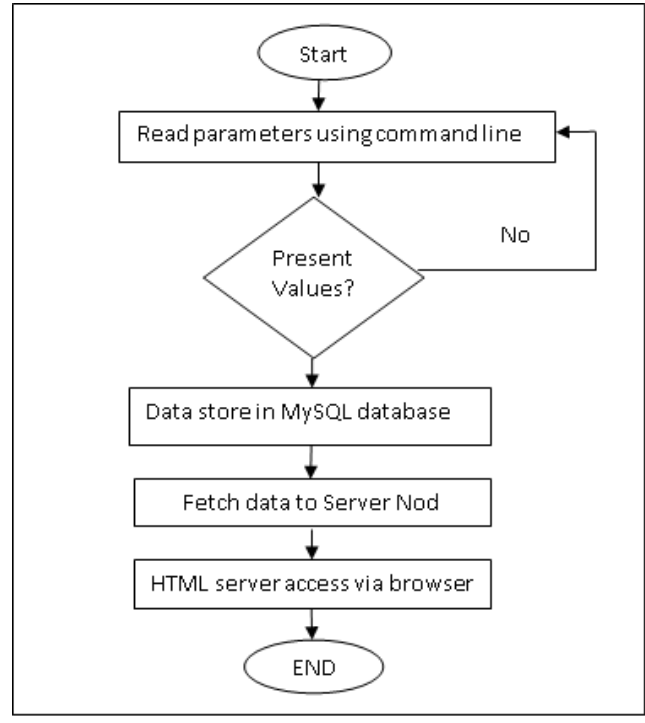

Figure 4. Flowchart of software programming operation

\section{PROOF-OF CONCEPT RESULTS}

The proposed wireless monitoring system designed a simple and clean web-based interface to display the data collected by multiple sensor nodes. The measured values are displayed in accordance with the settings, and the content can be viewed from user equipment such as laptops or smart phones. Figure 5 shows the website interface for the mart phones respectively. The sampling time interval are set to 1 minutes, therefore temperature sensor measured the water quality every one minute and stored the data into the local database. Then, the data is transferred to the server node automatically.

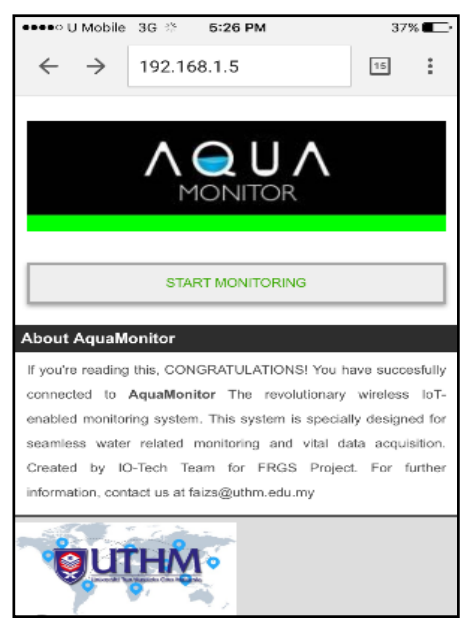

(a)

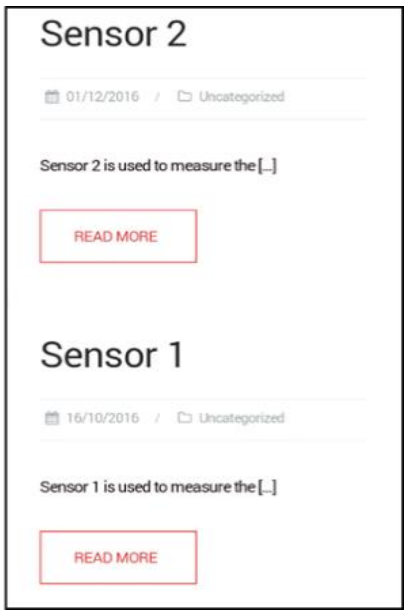

(b)

Figure 5. Web-based interface on a smartphone showing (a)Welcome page and (b) Sensor node selection

There are two sensor nodes deployed as a proof of concept and testing with a small fish ponds for their usability. Sensor node 1 is tested with a red tilapia pond while sensor node 2 is tested with a small fish pond. Both of the sensor node transferred the data to the server node based on SSH key-based authentication and mysqldump function. Mysqldump function is used to copy the database in sensor node to server node, while SSH key-based authentication is used to remote access to another microcomputer automatically without key in any password. Figure 6 shows the displays for sensor node 1 and 2. Notice at 6.26pm, Sensor 1 shows the temperature at $30^{\circ} \mathrm{C}$ and Sensor 2 shows $30.1^{\circ} \mathrm{C}$. This shows both sensor works simultaneously to sense two separate water environment. 


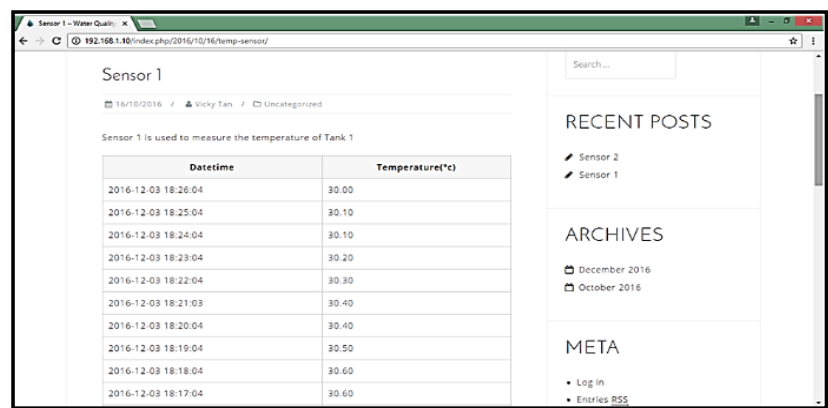

(a)

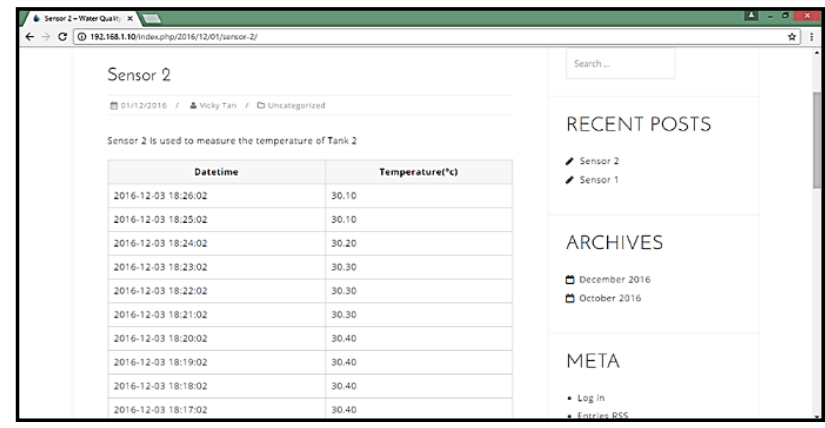

(b)

Figure 6. Real-time data from (a) Sensor node 1 and (b) Sensor node 2 simultaneously accessed via WiFi

The data is then can be made public to the world by using Internet of Things (IoT) platform such as Thingspeak, Carriot and Favoriot. Since this monitoring system have already local database capability, pushing the data to the cloud network is fairly easy. For instance, the data from server node is push to the Thingspeak channel and visualized as shown in Figure 7.

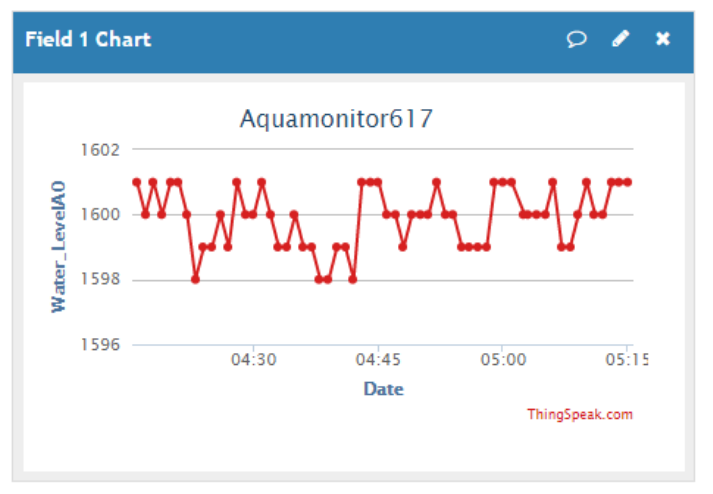

Figure 7. Thingspeak data visualization using the data provided by water quality monitoring system

\section{CONCLUSSION}

In this paper, we presented the design and implementation of a real-time wireless monitoring system for the use in high density aquaculture. The system is designed to provide continuously monitoring of water quality parameter in multiple tanks at the same time. The data generated by multiple sensor nodes are sent to the hybrid node for storage and processing then shared to multiple user via smartphones and laptops. The system is cost effective, easily deployable and user friendly compared with traditional monitoring methods used in aquaculture. The key design features in this system is the scalability where the number of nodes can be easily increased in a cluster topology because the system is designed to manage and transport data efficiently. 


\section{ACKNOWLEDGEMENTS}

This work was supported by Ministry of Higher Education (MOHE), Faculty of Engineering Technology, Office for Research, Innovation, Commercialization and Consultancy Management (ORICC), Universiti Tun Hussein Onn Malaysia (UTHM) under Short Term Grant (Vote No: U655). The system presented in this paper is registered intellectual property (PI2016704239).

\section{REFERENCES}

[1] "DRFT CWP Handbook on Aquaculture Statistics," vol. 3, no. October 2012, pp. 1-46, 2013.

[2] T. State and W. Fisheries, "The State of World Fisheries and Aquaculture 2014," 2014.

[3] Colt, John. "Water quality requirements for reuse systems." Aquacultural Engineering 34.3 (2006): 143-156.

[4] Zhang, Shi-Yang, et al. "An integrated recirculating aquaculture system (RAS) for land-based fish farming: The effects on water quality and fish production." aquacultural Engineering 45.3 (2011): 93-102.

[5] W. Sung, J. Chen, and H. Wang, "Remote fish aquaculture monitoring system based on wireless transmission technology," no. 57, 2014.

[6] D. S. Simbeye and S. F. Yang, "Water quality monitoring and control for aquaculture based on wireless sensor networks," J. Networks, vol. 9, no. 4, pp. 840-849, 2014.

[7] F. Regan, A. Lawler, and A. McCarthy, SmartCoast Project- Smart Water Quality Monitoring System, no. 30.2009.

[8] A. J. Odey, "AquaMesh - Design and Implementation of Smart Wireless Mesh Sensor Networks for Aquaculture," Am. J. Networks Commun., vol. 2, no. 3, p. 81, 2013.

[9] X. Zhu, D. Li, D. He, J. Wang, D. Ma, and F. Li, "A remote wireless system for water quality online monitoring in intensive fish culture," Comput. Electron. Agric., vol. 71, no. SUPPL. 1, 2010.

[10] D. He, D. Li, J. Bao, H. Juanxiu, and S. Lu, "A water-quality dynamic monitoring system based on web-serverembedded technology for aquaculture," IFIP Adv. Inf. Commun. Technol., vol. 346 AICT, no. PART 3, pp. 725-731, 2011.

[11] Jasman Bin Jaffar, "Development of a Water Temperature And pH Measurement system for Fish Ponds," 2009.

[12] S. S. S. , P. K. Sai Krishna Vaddadi, "Development of Embedded Wireless Network and Water Quality Measurement Systems for Aquaculture," pp. 637-641, 2012.

[13] H. L. Chen and X. Q. Liu, "A Novel Kind of Smart Monitoring System of Aquaculture," no. Icecee, pp. 369-372, 2015.

\section{BIOGRAPHIES OF AUTHORS}

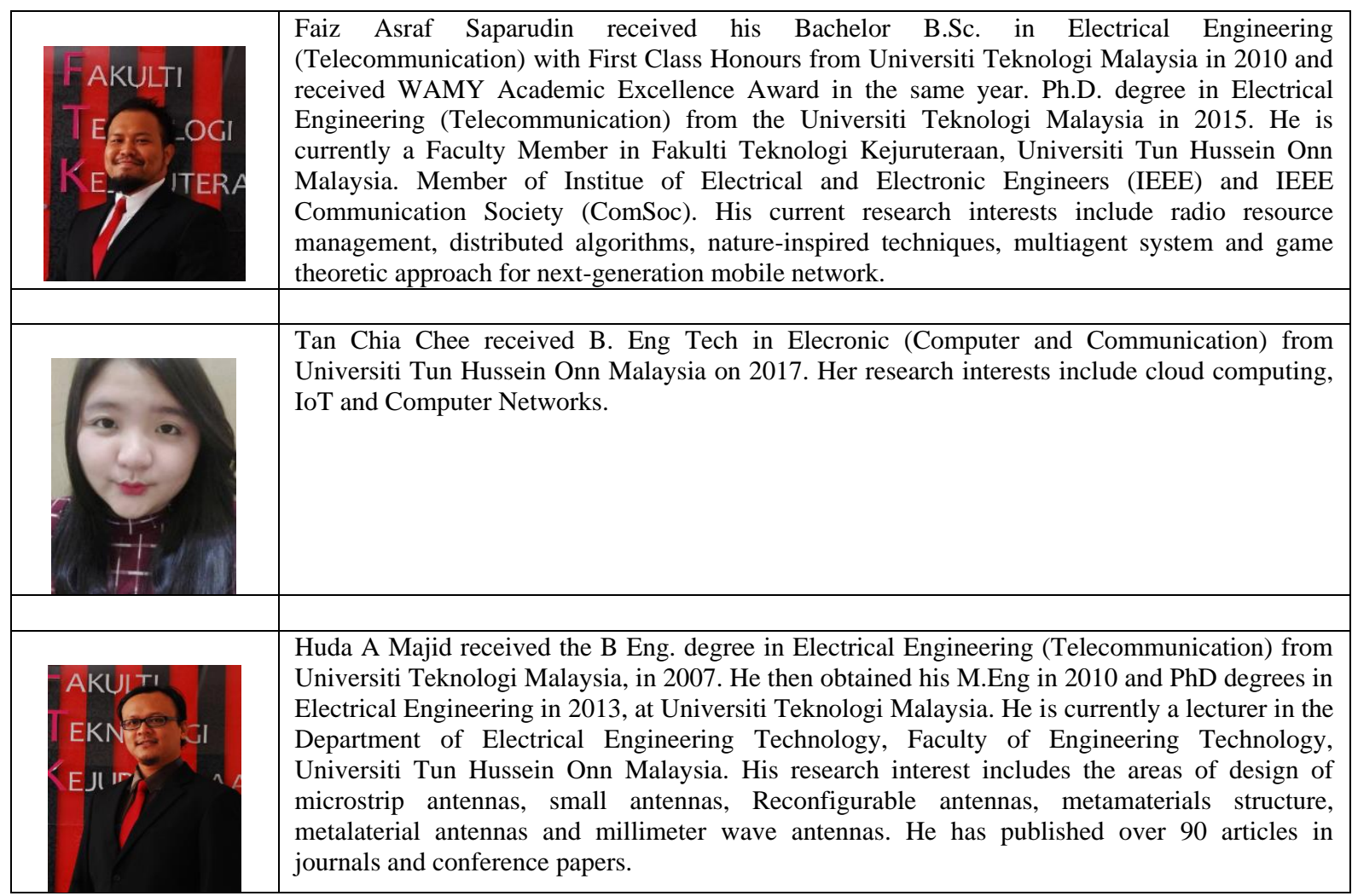

